Nervenarzt 2022 $\cdot 93: 392-401$

https://doi.org/10.1007/s00115-021-01165-2

Angenommen: 17. Mai 2021

Online publiziert: 3. August 2021

(c) Der/die Autor(en) 2021

\section{Ursachen visueller Halluzinationen bei der Parkinson-Krankheit}

\author{
Nico J. Diederich \\ Abteilung für Neurologie, Centre Hospitalier de Luxembourg, Luxemburg-Stadt, Luxemburg
}

Hintergrund: Visuelle Halluzinationen (VH) werden zumeist als Spätsymptome der Parkinson-Krankheit (PK) angesehen. Sie kommen jedoch in leichterer Form auch in Frühstadien der Erkrankung vor. Ursächlich wurden VH anfänglich als Folge einer dopaminergen Überstimulation gesehen, später ebenso im Rahmen einer demenziellen Entwicklung der PK.

Fragestellung: Die vorliegende Arbeit untersucht, ob sich das Entstehungsmodell der $\mathrm{VH}$ in den letzten Jahren erweitert hat.

Material und Methodik: Basierend auf klinischen, pharmakologischen und neuropathologischen Arbeiten sowie funktioneller Magnetresonanzgraphie erfolgt eine systematische Aufgliederung in monomodale und multimodale Entstehungsmodelle der VH. Die Anwendbarkeit auf unterschiedliche VH-Formen und -Auslösungsmomente wird jeweils kritisch beleuchtet.

Ergebnisse: Einbußen bei der visuellen Informationsaufnahme und -verarbeitung, Defizite der Aufmerksamkeit und fehlerhafte Konnektivität zwischen kortikalen Netzwerken werden herausgearbeitet. Es bestehen z. T. Überlappungen mit dem Lhermitte-Syndrom und dem Charles-Bonnet-Syndrom. Kein Modell erklärt jedoch befriedigend alle Spielarten der VH. Nicht alle VH weisen die gleiche Pathogenese und stets eine schlechte Prognose auf.

Schlussfolgerung: Die Ursachenkette visueller Halluzinationen ist komplex und individuell unterschiedlich. Inwieweit dies therapeutisch einsetzbar ist, ist bisher wenig erforscht. Es gibt erste Hinweise, dass neben einer Änderung der Medikation auch Visusverbesserung, die Einbindung des Partners/der Partnerin und vielleicht individuell anpassbare Copingstrategien erfolgreich eingesetzt werden könnten.

\title{
Schlüsselwörter
}

Parkinson-Krankheit · Visuelle Halluzinationen · Charles-Bonnet-Syndrom · Lhermitte-Syndrom . Copingstrategien

Visuelle Halluzinationen sind im späten Verlauf der Parkinson-Krankheit häufig und stellen eine Herausforderung für das Umfeld und den behandelnden Neurologen dar. Die zugrunde liegende Ursachenkette ist komplex und von Patient zu Patient unterschiedlich. Nicht nur die dopaminerge Medikation, sondern auch Aufmerksamkeitsstörungen, Vigilanzschwankungen und visuelle Defizite stellen Risikofaktoren dar. Bemerkenswerterweise setzen Patienten oft bereits ohne Fremdanleitung erfolgreich Copingstrategien ein.
Visuelle Halluzinationen (VH) gehören zu den häufigsten Spätkomplikationen der Parkinson-Krankheit (PK). Ihnen haftet der Ruf einer schlechten Prognose an, verbunden mit Einweisung ins Pflegeheim und höherer Mortalität [22]. Seit der Publikation einer Übersicht zu diesem Thema in Der Nervenarzt im Jahr 2003 [45] haben sich unsere Kenntnisse beträchtlich erweitert. Nicht nur kennen wir inzwischen weitere Spielarten der VH, sondern wir wissen auch, dass einige $\mathrm{VH}$-Formen frühzeitig im Krankheitsverlauf auftreten können. Auch ist erkannt worden, dass die Ursachenkaskade vielschichtiger und 
komplexer ist als ursprünglich angenommen. Außer der üblicherweise erwogenen Levodopa-Psychose [47] werden nämlich Visuseinbußen, ungünstige Lichtverhältnisse, Störungen des Schlaf-wach-Rhythmus [14] und neuerdings zunehmend subtile Veränderungen innerhalb kognitiver Netzwerke sowie deren gestörter Informationsaustausch untereinander $[50,58$, 62] ursächlich diskutiert. Kompensatorische Mechanismen können zur Anwendung kommen. Differenziertere Behandlungsstrategien, zugeschnitten auf das halluzinatorische Erleben des einzelnen Patienten, sind greifbarer geworden. Neben der üblichen Pharmakotherapie können auch nicht pharmakologische Strategien zur Anwendung kommen, wenngleich die Evidenzlage hier noch schwach ist. Im Gegensatz zu der früher stets als düster angesehenen Prognose der VH kann diese heute differenzierter und oft auch günstiger gestellt werden. Die vorliegende Arbeit vermittelt hierzu einen Überblick und gibt Ratschläge zur Umsetzung im Praxisalltag.

\section{Phänomenologie}

Fénelon und Mitarbeiter haben vor 20 Jahren erstmals auf leichtere (,Minor ${ }^{\prime \prime}$ ))Formen der Halluzination, nämlich „Vorbeihuschen" und "Anwesenheit", hingewiesen [16]. Das halluzinatorische Erleben einer "Anwesenheit" ist sensu stricto keine $\mathrm{VH}$, da der Patient angibt, eine fremde Person stehe hinter ihm; er nimmt diese also außerhalb seines Gesichtsfeldes oder „extrakampin“ wahr. Die Halluzination des "Vorbeihuschens" wird vom Patienten zumeist als belanglos eingestuft. Er gibt sie oft auch erst auf wiederholtes Nachfragen an. Es kann sich um das flüchtige Vorbeigehen eines Menschen, das Vorbeifliegen eines Insekts oder die Wahrnehmung eines nicht identifizierbaren Schattens handeln [38]. Bemerkenswerterweise kommen solche leichteren Halluzinationen bereits bei unbehandelten "De-novo"-Patienten vor [51]. Die Illusion, also das Verkennen eines real wahrgenommenen Gegenstandes z. B. wird ein Strauch als Mensch erfasst -, ist häufig an ungünstige Licht- oder Visusverhältnisse gebunden. Im Grenzbereich zwischen Illusion und Halluzination findet sich die selektive Diplopie als zumeist sehr kurzzeitige, doppelte Wahrnehmung isolierter Objekte oder Personen [48, 61]. Die klassischen VH sind ausgestaltet (,formed $^{\prime \prime}$ ) und wiederholen sich nicht selten. Ihr Inhalt sind zumeist Tier- oder Menschengruppen; die emotionale Betroffenheit bleibt gering, ebenso der Ichbezug $[2,11]$. Die meisten Fragebögen, so z. B. die Skala der MDS-UPDRS-Teil-Skala, unterscheiden, ob die Einsicht gewahrt bleibt (Pseudohalluzinationen nach alter Terminologie) oder nicht. Manche VH werden vom Patienten als „interessant" erlebt, da sie den oft monotonen Tagesablauf bereichern [56]. Paranoides Verarbeiten der VH kommt eher selten vor. Die Themen Verfolgung, Bedrohung, Verarmung werden dann am häufigsten angegeben. Eine besonders belastende Spielart stellt das Capgras-Syndrom dar. Hierbei verliert der Patient die Anmutung der Vertrautheit einer bekannten Person, z. B. des Partners oder der Partnerin, wenngleich er dessen oder deren Physiognomie richtig erkennt. Infolgedessen ist er der Überzeugung, die gesehene Person sei ein Doppelgänger, der befremdlicherweise genau über ihn Bescheid wisse. Solche Verkennungssyndrome kommen aber auch bei anderen neurodegenerativen Prozessen vor, wenngleich häufiger bei Lewy-Körperchen-Erkrankungen [32]. Multimodale Halluzinationen, also visuell und auditorisch oder visuell und zönästhetisch, sind eher selten und werden hier nicht gesondert betrachtet [40]. Auch der Dermatozoenwahn mit ungünstiger Prognose soll hier nur am Rande angesprochen werden. Erwähnenswert ist hingegen, dass PK-Patienten und solche mit Lewy-Körperchen-Demenz das gleiche phänomenologische Spektrum der VH aufweisen [15, 46]. Wahneinfälle schließlich können eigenständig, also auch ohne VH, auftreten. Verfolgt und Bestohlen werden bilden übliche Themen. Das Othello-Syndrom kennzeichnet eine wahnhafte Eifersucht, und dem Partner bzw. der Partnerin wird dabei eine sexuelle Beziehung mit anderen unterstellt. Ein häufigeres Vorkommen bei eher jüngeren Männern, insbesondere unter Therapie mit Dopaminagonisten, wurde in diesem Zusammenhang beschrieben ([35]; • Tab. 1).

\section{Häufigkeit}

Nicht alle Patienten berichten spontan über ihre $\mathrm{VH}$, vielfach muss mehrfach nachgefragt werden [24]. Dies gilt nicht nur für die oben beschriebenen klassischen $\mathrm{VH}$, sondern insbesondere auch für die Halluzinationen der "Anwesenheit" und des "Vorbeihuschens". Frühe Querschnittsstudien haben eine Häufigkeit von 15-38\% für komplexe VH ermittelt [1]. In Bezug auf die leichteren Halluzinationen wird sogar von einer Häufigkeit von $42 \%$ bei De-novo-Patienten und von $50 \%$ bei behandelten Patienten berichtet [51, 64]. Hier ist allerdings Vorsicht geboten, da diese Zahlen noch nicht bei größeren Kohorten bestätigt worden sind. Bei einer groß angelegten Langzeitstudie über 12 Jahre betrug die Häufigkeit der $\mathrm{VH}$ sogar $60 \%$ [20]. Es ist möglich, dass in einem fortgeschrittenen Stadium der PK mit ausgeprägter Demenz die „kreative“ halluzinatorische Produktion wieder verblasst, in etwa vergleichbar der zunehmenden Anosognosie für mnestische Defizite bei fortgeschrittener Demenz. Allerdings ist dies bisher noch nicht statistisch belegt.

\section{Pathologie}

Es waren als erstes Arbeiten zur Neuropathologie, die auf unterschiedliche Entstehungsmöglichkeiten der VH hinwiesen [27, 33]. Bei früh im Verlauf auftretenden $\mathrm{VH}$ finden sich Lewy-Körper (LK) in den Amygdala, insbesondere im basolateralen Kern und im Parahippokampus; bei späterer VHManifestation finden sich hingegen LK im Claustrum und Okzipitalkortex, in Verbindung mit einer Atrophie dieser Areale. Eine Hirnatrophie konnte auch in anderen Regionen nachgewiesen werden, u.a. in der oberen und lateralen Parietalregion und im lateralen Frontalkortex [18, 54]. Kasuistisch wurde bei Patienten mit Lewy-Körper-Demenz (LKD) auch über einen LK-Nachweis in der Retina berichtet [43]. Es ist bemerkenswert, dass bei Synukleinopathien mit VH autoptisch auch Amyloidablagerungen und neurofibrilläre Tangles und geminderte Konzentrationen von Amyloid $A \beta$ 1-42 im Liquor nachweisbar sind [30]. Bislang fehlen allerdings systematische Studien zum Krankheitsbefall von Hirnregionen, die für Vigilanz, Aufmerksamkeit und 


\section{Übersichten}

Tab. 1 Formen von Halluzinationen bei der Parkinson-Krankheit

\begin{tabular}{|c|c|c|}
\hline Symptom & Beschreibung & Ursache und Vorkommen \\
\hline $\begin{array}{l}\text { Gefühl der Anwesen- } \\
\text { heit („sensation de pré- } \\
\left.\text { sence }{ }^{\prime \prime}\right) \\
\text { Extrakampine Halluzina- } \\
\text { tion }\end{array}$ & $\begin{array}{l}\text { Eindruck eine andere, ungefährliche Person stehe } \\
\text { hinter dem Patienten }\end{array}$ & $\begin{array}{l}\text { Ungeklärt } \\
\text { Zusammenhang mit REM-Schlafstörung diskutiert } \\
\text { Bereits bei De-novo-PK-Patienten beschrieben } \\
\text { Ist eher selten }\end{array}$ \\
\hline $\begin{array}{l}\text { Flüchtiges Vorbeihu- } \\
\text { schen ("sensation de } \\
\text { passage") }\end{array}$ & $\begin{array}{l}\text { Flüchtiger Eindruck, eine Person oder ein Schatten be- } \\
\text { finde sich im peripheren Gesichtsfeld oder ein Vogel/ } \\
\text { Insekt fliege vorbei; Patient erkennt zumeist sofort die } \\
\text { Unwirklichkeit, berichtet darüber erst auf Anfrage }\end{array}$ & $\begin{array}{l}\text { Ungeklärt } \\
\text { Fehlinformation/Fehlinterpretation des visuellen Inputs } \\
\text { Vorkommen ausschließlich im peripheren Gesichtsfeld } \\
\text { Bereits bei De-novo-PK-Patienten beschrieben } \\
\text { Ist häufig, muss aber erfragt werden }\end{array}$ \\
\hline Visuelle Illusion & $\begin{array}{l}\text { Tatsächlich wahrgenommenes Objekt wird fehlinter- } \\
\text { pretiert }\end{array}$ & $\begin{array}{l}\text { Fehldeutung der Sinnhaftigkeit („salience”) } \\
\text { Mangelhafte Lichtverhältnisse („Dämmerung“) oder emotiona- } \\
\text { le Faktoren (Angst usw.) können das Auftreten begünstigen } \\
\text { Ist häufig }\end{array}$ \\
\hline $\begin{array}{l}\text { Sonderform der visuellen } \\
\text { Illusion: Pareidolie }\end{array}$ & $\begin{array}{l}\text { Fehldeutung eines leblosen Objektes als bedrohliches } \\
\text { Lebewesen }\end{array}$ & $\begin{array}{l}\text { Möglicher Hinweis auf Lewy-Körperchen-Demenz } \\
\text { Ist eher selten }\end{array}$ \\
\hline $\begin{array}{l}\text { Optische Halluzinationen } \\
\text { im frontalen Gesichtsfeld }\end{array}$ & $\begin{array}{l}\text { Menschen- oder Tiergruppe, oft repetitiv, oft nur flas- } \\
\text { hartig auftretend, ohne Ichbezug }\end{array}$ & $\begin{array}{l}\text { Unterschiedliche Ursachen, z. B. dopaminerge } \mathrm{D}_{3}-\text { und } \mathrm{D}_{4}{ }^{-} \\
\text {Überstimulation; anticholinerge Blockierung; fehlerhafter } \\
\text { visueller Input; Aufmerksamkeitsstörung } \\
\text { Ist häufig }\end{array}$ \\
\hline Selektive Diplopie & $\begin{array}{l}\text { Einzelobjekt oder Einzelperson wird doppelt wahrge- } \\
\text { nommen }\end{array}$ & $\begin{array}{l}\text { Unterschiedliche Mechanismen: z. B. Heterophorie; mangelhaf- } \\
\text { te Fusion der retinal generierten Bilder; Aufmerksamkeitsstö- } \\
\text { rung } \\
\text { Ist eher selten }\end{array}$ \\
\hline $\begin{array}{l}\text { Akustische Halluzinatio- } \\
\text { nen }\end{array}$ & $\begin{array}{l}\text { Selten isoliert; zumeist assoziiert mit visuellen Halluzi- } \\
\text { nationen }\end{array}$ & $\begin{array}{l}\text { Idem } \\
\text { Fördernder Einfluss durch Presbyakusis möglich } \\
\text { Ist eher selten }\end{array}$ \\
\hline $\begin{array}{l}\text { Taktile Halluzinationen } \\
\text { („Dermatozoenwahn“) }\end{array}$ & $\begin{array}{l}\text { Perzeption eines unangenehmen Hautkontakts, z. B. } \\
\text { durch Insekten usw. }\end{array}$ & $\begin{array}{l}\text { Oft wahnhafte Deutung } \\
\text { Vorkommen bei demenzieller Entwicklung } \\
\text { Ist sehr selten }\end{array}$ \\
\hline Capgras-Syndrom & $\begin{array}{l}\text { Bekannte Person (bes. Partner) wird als Doppelgänger } \\
\text { betrachtet }\end{array}$ & $\begin{array}{l}\text { Physiognomie wird richtig erkannt, Gefühl der Familiarität ist } \\
\text { jedoch verloren } \\
\text { Störung zwischen striatalem visuellem Kortex und limbischem } \\
\text { System } \\
\text { Ist selten }\end{array}$ \\
\hline Wahneinfälle & $\begin{array}{l}\text { Beziehen sich oft auf VH, können aber auch isoliert } \\
\text { auftreten } \\
\text { Verfolgung und Diebstahl sind Hauptthemen }\end{array}$ & $\begin{array}{l}\text { Ungeklärt } \\
\text { Oft Vorzeichen der demenziellen Entwicklung } \\
\text { Ist eher selten }\end{array}$ \\
\hline $\begin{array}{l}\text { Sonderform der Wahn- } \\
\text { einfälle: Othello-Syn- } \\
\text { drom }\end{array}$ & Wahnhafte Eifersucht & $\begin{array}{l}\text { Besonders bei jüngeren Männern } \\
\text { Mögliche Nebenwirkung der Dopaminagonisten? } \\
\text { Ist sehr selten }\end{array}$ \\
\hline
\end{tabular}

visuelle Verarbeitung verantwortlich sind. Da zudem die meisten Autopsien bei Patienten im Spätstadium durchgeführt werden, könnten Atrophie und LK-Befall auch durch allgemeines Fortschreiten der Erkrankung und Alterungsprozesse erklärbar sein. Deshalb sind die Daten von in vivo durchführbaren Bildgebungsverfahren ggf. sogar während des Erlebens einer $\mathrm{VH}$ als spezifischer anzusehen. Solche Befunde werden weiter unten angeführt.

\section{Monomodale Modelle}

Der klinische Alltag suggeriert vordergründig monomodale Erklärungsmodelle der VH. Handelt es sich nicht einfach um eine dopaminerge Psychose, früher auch Levodopapsychose genannt, da die Reduktion dieser Medikation oft Erleichterung bringt? Da VH gehäuft nachts auftreten, wurde spekuliert, ob eine Verbindung mit gestörtem REM-Schlaf bestehen könnte [3]. Auch wurde postuliert, dass eine Dysfunktion des visuellen Systems eine entscheidende Rolle spielen könnte. Allerdings decken bei näherer Analyse diese "simplen“ Er- klärungsmodelle nicht das gesamte Spektrum der VH ab. Daher sind eine komplexere Ursachenverflechtung, vielleicht aber auch unterschiedliche Entstehungsweisen für unterschiedliche Arten der VH zu erwägen. Die wichtigsten monomodalen ätiologischen Hypothesen werden trotzdem kritisch im Folgenden dargestellt, um später auch die multimodalen Modelle der Entstehung besser verstehen zu können.

\section{Die dopaminerge Psychose}

Die Arbeitsgruppe um Harald Klawans und Christopher Goetz postulierte in den 
1970er-Jahren, dass die kontinuierliche dopaminerge Medikation zur Überstimulation mesolimbischer D3- und D4Rezeptoren führt [47]. Die Auslösung eines „Kindling"-Phänomens wäre die Folge: Dopaminrezeptoren würden nach längerer Therapiedauer überempfindlicher, sodass selbst geringe Dopamindosen VH auslösen könnten. Auch wurde ein Ungleichgewicht verschiedener Neurotransmitter diskutiert. Insbesondere bei der LKD weisen quantitative neurochemische Autopsiebefunde auf einen Verlust cholinerger Neurone im Neokortex hin. Geht deren Filterfunktion bezüglich nicht relevanter interner oder externer sensorischer Informationen verloren, so werden diese Informationen nicht mehr als "Hintergrundrauschen" verarbeitet, sondern als $\mathrm{VH}$ in die bewusste Wahrnehmung aufgenommen [52]. Ebenso könnte die Levodopatherapie einen relativen Serotoninmangel verursachen und sekundär eine postsynaptische Serotoninrezeptorüberempfindlichkeit hervorrufen. Neurotoxikologische Befunde nach LSDEinnahme legen hier eine besondere Rolle des 5-HT2A-Rezeptors nahe [41]. So wurde bei PK-Patienten mit VH über eine reduzierte postsynaptische Darstellung dieses Rezeptors berichtet [4].

Im klinischen Alltag hat sich die Anwendbarkeit dieser Transmitterhypothesen bewährt. Die Reduktion von Medikamenten mit anticholinerger Wirkung, die Reduktion der dopaminergen Medikation, besonders der DA-Agonisten mit D3- und D4-Angriffspunkt, oder auch der Einsatz von Clozapin und Pimavanserin, die u. a. als Antagonisten des 5-HT2A-Rezeptors agieren, können die VH reduzieren. Der Einsatz der atypischen Neuroleptika wurde im Detail in Der Nervenarzt beschrieben [28].

Jedoch gibt es auch zahlreiche Argumente, die diese "Monopolstellung" der pharmakologischen Erklärungsmodelle infrage stellen. So wurde bereits vor der Levodopaära über VH bei PK-Patienten berichtet [17]. Die Halluzinationen des „Vorbeihuschens" und der "Anwesenheit" sind auch bei unbehandelten De-novo-PK-Patienten nachweisbar [51]. Ebenso berichten LKD-Patienten über VH auch ohne dopaminerge Medikation [15, 46]. Nicht zuletzt scheiterte ein VH-Triggerversuch: PK-Patienten, die zu Hause häufig VH erlebten, berichteten erstaunlicherweise nicht über VH bei intravenöser Applikation einer hohen Levodopadosis im Labor [23].

\section{VH als Traumintrusion in den Wachzustand}

Im Jahr 1922 berichtete Jean Lhermitte [39], dass Patienten mit pedunkulären Hirnstammläsionen insbesondere vaskulärer Natur über geformte, farbige und sehr lebhafte VH klagten, die sie häufig auch wahnhaft verarbeiteten. Diese Patienten wiesen deutliche Störungen des Schlafwach-Rhythmus auf. Lhermitte charakterisierte den prototypischen Patienten mit pedunkulären Halluzinationen als einen „wachen oder nur unvollständig schlafenden Träumer". Erst viel später wurde die Schlüsselfunktion des dorsolateralen $\mathrm{Nu}$ cleus geniculatus erkannt [41]. Üblicherweise unterliegt diese Struktur dem hemmenden Einfluss der Raphé-Kerne und des Nucleus pedunculopontinus (NPP). Beim Fehlen dieser Hemmung kommt es zur ungebremsten Erregung verschiedener Thalamuskerne und des Kortex. Im Falle der PK könnte ein solcher Mechanismus tatsächlich aktiv werden, da bereits in vivo eine Atrophie des NPP nachweisbar ist und die Autopsie einen zusätzlichen LK-Befall dieses Kerns anzeigt [31, 57]. Einschränkend ist jedoch festzustellen, dass PK-Patienten zumeist keine Unterschiede zwischen Tagund Nachtzeit in Bezug auf Erscheinungsformen der VH aufweisen. Mittels Polysomnographie konnte aber ein Auftreten der VH unmittelbar im Anschluss an eine REMSchlaf-Phase beobachtet werden [3]. Auch legen Korrelationsstudien einen Zusammenhang zwischen VH und REM-SchlafVerhaltensstörung nahe [1]. Es bestehen also Gemeinsamkeiten von einerseits dem Lhermitte-Syndrom und andererseits den VH bei der PK. So ist in beiden Fällen eine Intrusion von REM-Schlaf-Fragmenten in den Wachzustand zu postulieren; die Neuropathologie deutet in beiden Fällen auf Läsionen der "Traumfabrik" und der Schlafregulierungszentren hin [49]. Doch auch diese Hypothese kann nicht sämtliche VH erklären. So weisen z.B. die VH des PK-Patienten nicht die bizarren Charakteristika des REM-Schlafs auf. Zudem erleben PK-Patienten VH auch bei vollem Bewusstsein.

\section{VH infolge visueller sensorischer} Deprivation

Charles Bonnet beschrieb VH bei seinem unter einer Katarakt leidenden, aber geistig noch regen Großvater. Der ältere Herr hatte komplexe, stereotype, sich oft wiederholende $\mathrm{VH}$, die plötzlich auftraten und ebenso rasch wieder verschwanden. Seitdem beschreibt das Charles-Bonnet-Syndrom solche flashartig auftretenden $\mathrm{VH}$ bei älteren Patienten mit zumeist reduzierter Sehkraft und gelegentlich auch eingeschränkten kognitiven Funktionen [59]. PK-Patienten weisen unterschiedliche Einbußen des Sehens hinsichtlich Kontrasterkennen und Farbdiskrimination auf [2, 53]. Ein Zusammenhang mit dem Auftreten von VH konnte sowohl bei nicht dementen PK-Patienten als auch bei LKDPatienten nachgewiesen werden [10, 42]. Die Verarbeitung des visuellen Inputs ist ebenso gestört. PK-Patienten mit VH benötigen eine längere Reaktionszeit als PKPatienten ohne $\mathrm{VH}$, um einen visuellen Input bewusst wahrzunehmen [37]. Die aufsteigende („,bottom-up“) visuelle Verarbeitung ist gestört. Mit funktioneller Magnetresonanztomographie wurde nachgewiesen, dass bei PK-Patienten mit VH vor dem bewussten Bilderkennen Areale des Okzipitalkortex, des Gyrus fusiformis und der Frontalregion weniger ausgeprägt und verzögert aktiviert werden, als dies bei PKPatienten ohne VH der Fall ist [44]. Die verminderte Aktivierung des Gyrus fusiformis und des Gyrus lingualis hält auch während des Bilderkennens an. Diese Befunde deuten auf eine ursächlich wirkende partielle visuelle Deprivation hin, ähnlich wie dies beim Charles-Bonnet-Syndrom der Fall ist [36]. Der qualitativ mangelhafte visuelle Input ermöglicht das Auftauchen („popping up") gespeicherter oder sui generis generierter Bilder in das Bewusstsein. Strenge Gütekriterien bezüglich der perzeptuellen Wirklichkeit eines Inputs sind damit aufgegeben [7].

In den letzten Jahren häuften sich die Befunde in Bezug auf den mangelhaften visuellen Input bei der PK [26, 63]. PK-Patienten mit VH weisen eine dünnere retinale Nervenfaserschicht auf als PK-Patienten ohne VH [36], möglicherweise sekundär nach Befall der retinalen ganglionären Zellschicht [34]. Beim Vorliegen von VH zeigt 


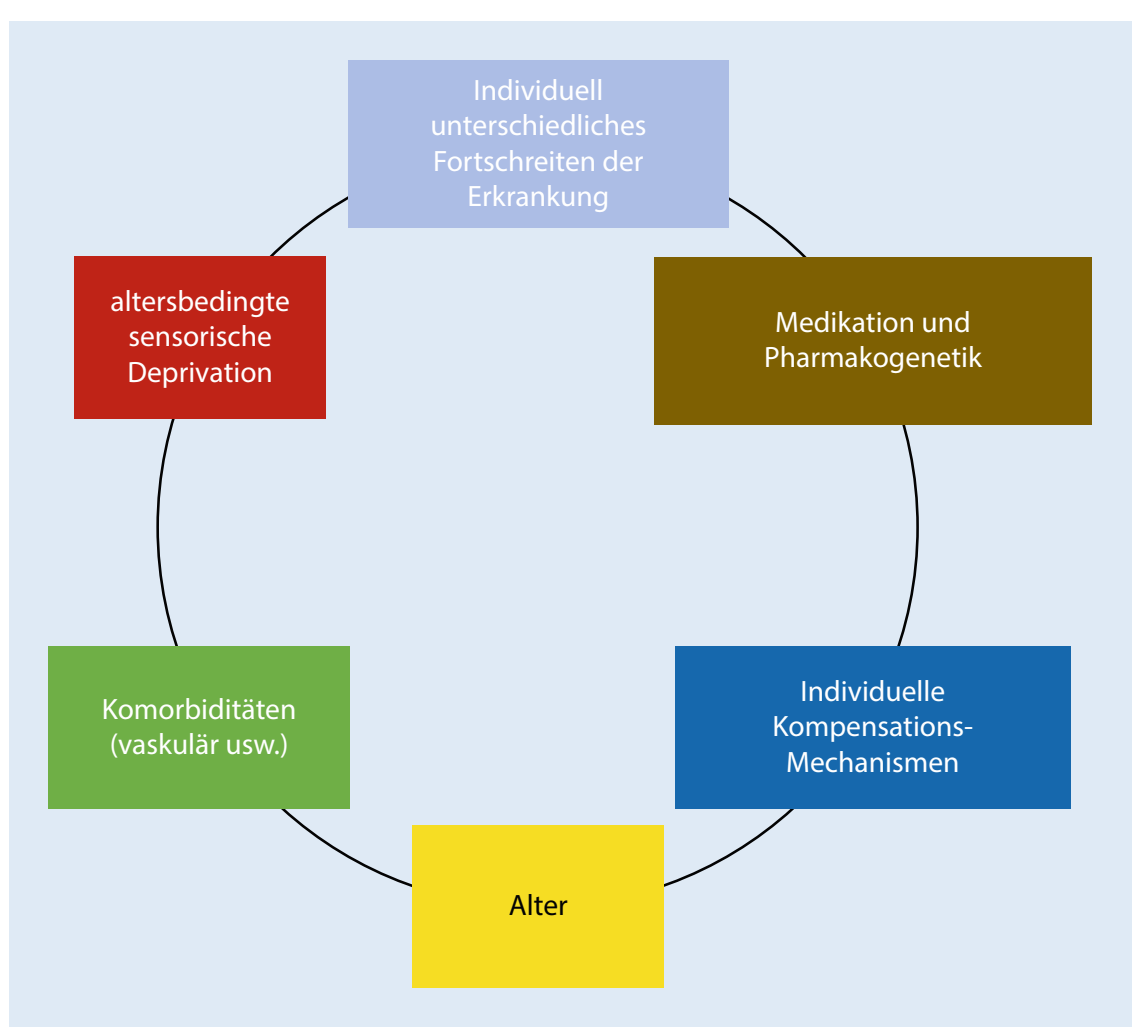

Abb. 1 A Allgemeine Faktoren, die das Ausmaß dervisuellen Halluzinationen bei der Parkinson-Krankheit beeinflussen

die Magnetspektroskopie eine reduzierte GABA-Konzentration im primären visuellen Kortex. Da reduzierte GABA-Spiegel die visuelle Verarbeitung eines bruchstückhaften Inputs "glätten“, kommt es häufiger zur Fehlinterpretation von mehrdeutigen Inputs [19].

Das Konzept des Charles-Bonnet-Syndroms erklärt nur teilweise VH bei der PK. Zwar ist in beiden Szenarien der primäre visuelle Input mangelhaft und die emotionale Betroffenheit gering. Auch kann die Einsicht gewahrt bleiben. Im experimentellen Setting jedoch vermag Flackerlicht bei PK-Patienten mit intakter Aufmerksamkeit lediglich nichtfigürliche VH hervorrufen, nicht aber VH von lebenden Gestalten [65]. Das Modell liefert auch keine Erklärung für sekundäre paranoide Deutungen.

\section{Multimodale Modelle (• Abb. 1, 2 und 3)}

Wie gezeigt, sind die monomodalen Erklärungsversuche zwar attraktiv, da einfach zu belegen und oft klinisch auch sofort anwendbar. Jedoch vermag kein Modell die individuell unterschiedliche Suszeptibilität
- das Modell zur Aufmerksamkeitskontrolle,

- die Hypothese der thalamokortikalen Dysregulation (TKD).

Die Modelle werden in der Chronologie ihrer Veröffentlichung vorgestellt.

\section{Das AIM-Modell nach Hobson}

Dies ist ein dreidimensionales Modell [13]. Die Aktivierung stellt die erste Achse dar; sie reicht vom tiefen NREM-Schlaf über REM-Schlaf bis zum Wachzustand. Der Input beschreibt den Informationsaustausch mit der Außenwelt. Letzterer schwankt abhängig davon, ob die „Schleusen zur Außenwelt geöffnet oder geschlossen" sind. Das System lässt aber eine gewisse „Porosität" zu. Die Modulation integriert über die Zeitachse aminerge (Noradrenalin und Serotonin) und cholinerge Einflüsse und beschreibt außerdem den möglichen Einfluss einer Medikation. Unregelmäßigkeiten können in diesem Modell sowohl die A-Achse (reduzierte Vigilanz), die I-Achse (geminderte Stärke des visuellen Inputs) oder ebenso die M-Achse (Ungleichgewicht der Transmitter und Medikamenteneinfluss) betreffen. Störungen berühren auch das Filtern externer Bilder bzw. die Generierung interner Bilder [13]. Die multifaktorielle Genese der VH, welche das Modell vorschlägt, konnte in einer Kohorte von 84 PK-Patienten, davon ein Drittel mit VH und zwei Drittel ohne VH, bestätigt werden [21]. So wurden als VH-bestimmende Faktoren multimodal u. a. die visuelle Perzeptionsstärke, kognitive Einbußen sowie eine REM-Schlaf-Verhaltensstörung erkannt. Die praktische Anwendbarkeit des AIM-Modells ist also gegeben. Geminderte Inputstärke z. B. bei Dämmerlicht bewirkt illusionäre Verkennungen eines Gegenstandes; Intensitätszunahme der intern generierten Bilderführt zur REM-Intrusion in den Wachzustand; mangelhafter visueller Input mit gleichzeitiger Akzeptanz intern gebildeter Bilder produziert VH in normaler Umgebung [13]. Auch für die selektive Diplopie werden sich überlappende Mechanismen diskutiert, so z.B. Heterophorie bei Ermüdung als auch reduzierte Aufmerksamkeit oder mangelhafte Fusion der beidseits retinal generierten Bilder [48, 61]. Das Modell kann somit zwar VH un- 

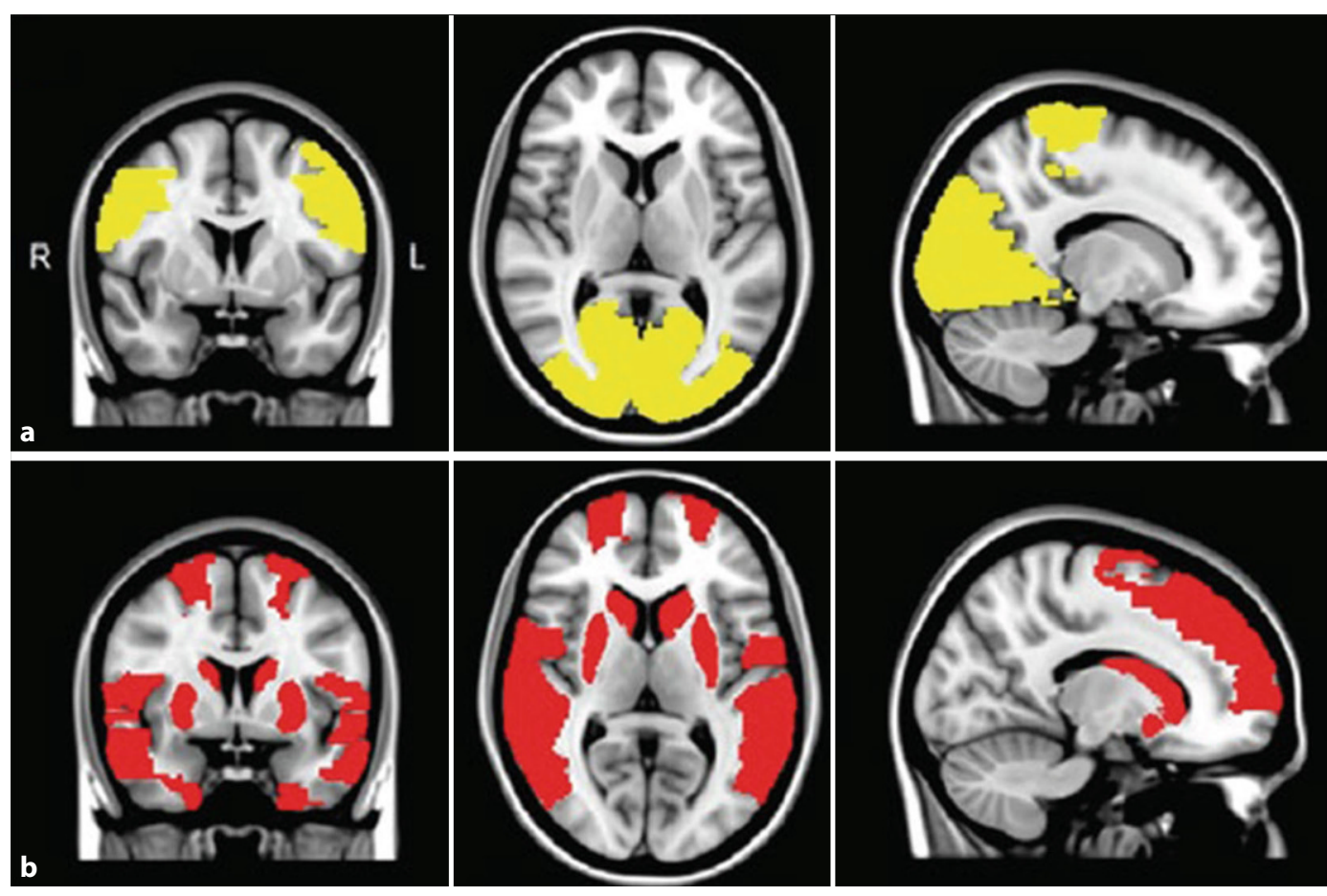

Abb. $2 \varangle$ Hirnareale mit geminderter funktioneller Konnektivität bei Parkinson(PK)-Patienten mit visuellen Halluzinationen (VH). Magnetresonanztomographische Bilder zeigen verminderte funktionelle Konnektivität (FK) im Vergleich zu gesunden Probanden. a PK-Patienten insgesamt, b PK-Patienten mit VH.PK-Patienten weisen insgesamt verminderte FK in parazentralen und okzipitalen Arealen (gelb) auf. PK-Patienten mit VHzeigen zusätzlich verminderte FK in frontalen, temporalen und subkortikalen Arealen (rot). Bezüglich Methodik siehe [29]. (C) The Radiological Society of North America (RSNA). Alle Rechte vorbehalten. Abdruck mit freundl. Genehmigung)

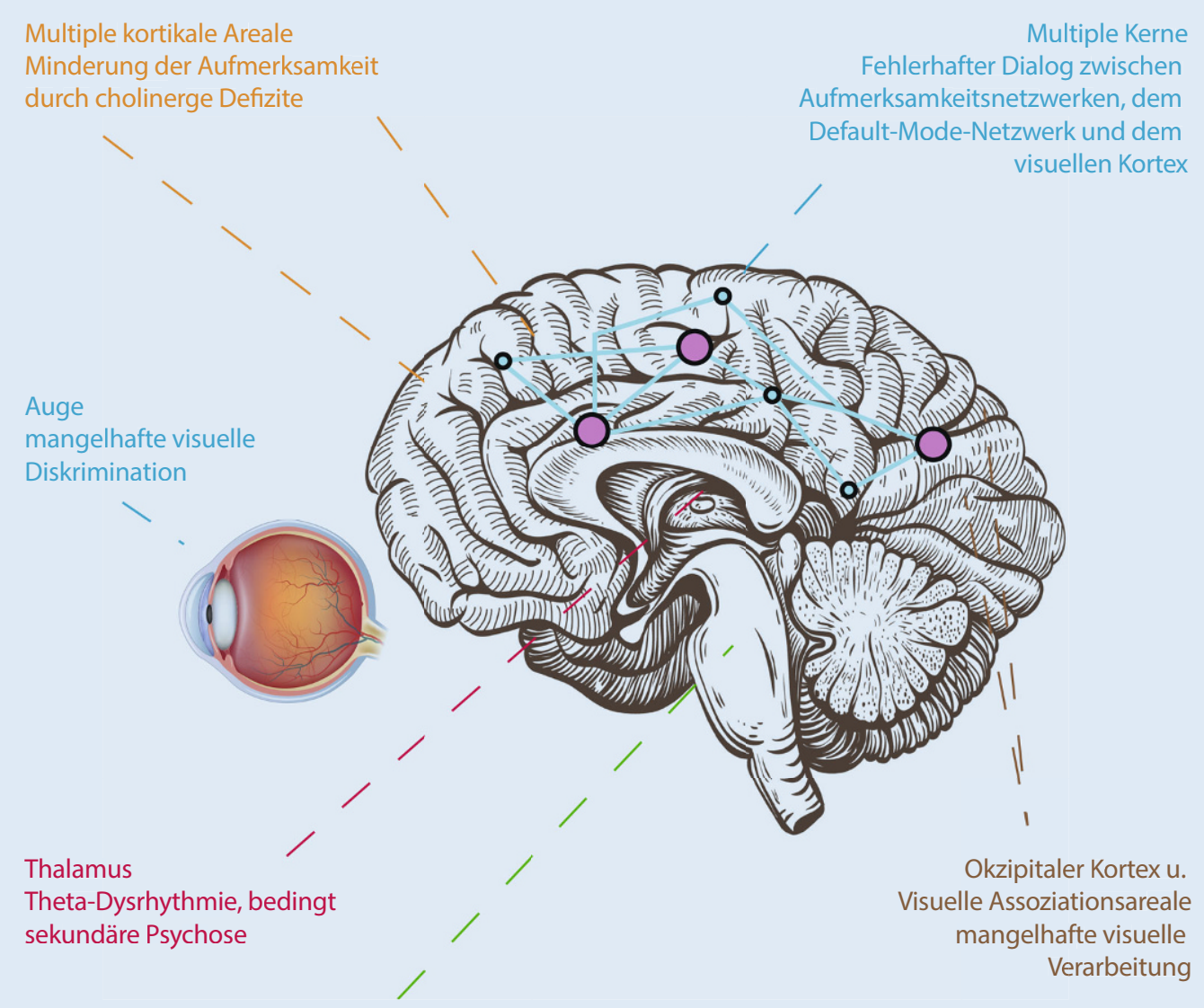

Nucleus Pedunculopontinus

Störung des Schlaf-Wach-Rhythmus und REM-Intrusion in Wachzustand
Abb. $3 \varangle$ Multiple, z. T. überlappende Ursachen visueller Halluzinationen bei der Parkinson-Krankheit 
ter sehr unterschiedlichen Bedingungen erklären; es vermag jedoch weder deren sich wiederholenden Inhalt noch die Entwicklung einer paranoiden Psychose zu erklären.

\section{Das Modell der Perzeptions- und Aufmerksamkeitsdefizite}

In Bezug auf die visuelle Wahrnehmung trennt dieses Modell klar die Ebenen „bottom up" und "top down" [9]. Liefert erstere Ebene einen qualitativ mangelhaften visuellen Input, so treten sog. „Proto-Objekte“ unbewusst als Ersatz in Erscheinung. Sie werden teils neu generiert, teils aus dem Gedächtnisspeicher abgerufen. Bei geminderter Aufmerksamkeit werden sie unkritisch als reale Objekte akzeptiert, sprich als VH wahrgenommen. Dieses Modell stellt geminderte Aufmerksamkeit als entscheidenden Faktor bei der VH-Entstehung in den Vordergrund. Die Minderung kann verursacht werden durch kortikale cholinerge Defizite [52] oder auch durch die Einnahme von Anticholinergika. Das Modell vermag aber weder den VH-Inhalt zu erklären noch die klinische Entwicklung der VH vorherzusagen.

\section{Das Modell zur Aufmerksamkeits- kontrolle}

Bedingt durch die rasante Entwicklung der funktionellen Magnetresonanztomographie (fMRT) ist das Modell zur Aufmerksamkeitskontrolle am besten experimentell belegt [58]. Es legt zuerst dar, dass normalerweise das ventrale Aufmerksamkeitsnetzwerk („,ventral attention network") die Bedeutung oder Sinnhaftigkeit (,salience“) eines vorerst noch nicht gedeuteten Inputs überprüft. Hierzu ist ein „Dia$\log ^{\prime \prime}$ mit dem im Ruhezustand überwiegenden "Default-mode"-Netzwerk erforderlich. Erst dann erkennt schließlich das dorsale Aufmerksamkeitsnetzwerk korrekt den visuellen Input. Bei PK-Patienten mit VH funktionieren diese "Zwiegespräche“ zwischen den einzelnen Netzwerken nicht mehr und es kommt zu Fehlbestimmungen, so z. B. zur illusionären Verkennung eines Gegenstandes. Dieses Modell kann besonders die Entstehung von Illusionen gut erklären. Abnorm gesteigerte Konnektivität zwischen dem „Default-mode“-Netz- werk und dem visuellen Kortex könnte begründen, warum „Gedankenabschweifen" (",mind wandering") bei PK-Patienten mit $\mathrm{VH}$ ausgeprägter ist als bei solchen ohne VH [62]. Eine fehlerhafte Konnektivität zwischen den Netzwerken wird auch bei der Entstehung der leichteren VH angenommen [6] und dürfte damit generell einen früh einsetzenden pathophysiologischen Mechanismus bei der PK darstellen. Letztlich ist es aber fast unmöglich, sämtliche beobachtete Minderungen der funktionellen Konnektivität (• Abb. 2; [29]) in ein einziges Modell zu integrieren.

\section{Die thalamokortikale Dysrhythmie}

Die jüngste Hypothese postuliert einen Zusammenhang zwischen der thalamokortikalen Dysrhythmie (TKD) und VH bei Patienten mit Parkinson-Demenz oder mit LKD [49]. Entsprechend dieser Annahme hemmt die in Thalamuskernen generierte Theta-Dysrhythmie frontale Aufmerksamkeitsnetzwerke und führt damit zur Entkopplung mit dem „Default-mode"-Netzwerk. Dessen Eigenaktivität wird nun nicht mehr kontrolliert und führt zu willkürlichen Verbindungen zwischen autobiografischen Erinnerungsfragmenten und dem noch zu identifizierenden visuellen Input. Dies wiederum bedingt illusionäre Verkennungen, z.B. von Gegenständen als bekannte Personen (Pareidolien), und schließlich wahnhafte Interpretationen des Erlebten. Die Autoren sehen auch einen Zusammenhang zwischen der TKD und ähnlichen Theta-Rhythmen im REMSchlaf von PK-Patienten. Sie bestätigen damit indirekt die Hypothese der VH als REM-Intrusion in den Wachzustand. Den posterioren Thalamuskernen und dem posterioren zingulären Kortex kommt hierbei entscheidende pathogenetische Bedeutung zu, da diese Areale LK-beladen sind und/oder atrophisch geworden sind. Zudem unterliegen sie bei der PK der bereits oben erwähnten fehlerhaften Stimulation durch den NPP. Dieses Modell erscheint am besten geeignet, um das sekundär-paranoide Erleben zu erklären, ist aber ebenso wie die vorher aufgeführten Modelle ungeeignet, alle Spielarten der VH zu erklären.
Eine Synthese im Bild der wichtigsten $\mathrm{VH}$-induzierenden Faktoren wird in - Abb. 3 vorgeschlagen.

\section{Kompensatorische Mechanismen}

Diese Übersicht geht nicht auf die Pharmakotherapie der VH ein, da diese kürzlich und erschöpfend in Der Nervenarzt erörtert worden sind [28]. Der Streifzug durch monomodale und multifaktorielle Erklärungsmodelle mag manchem Kliniker theoretisch und praxisfern vorgekommen sein. Bei näherer Betrachtung zeigt sich allerdings, dass diese Modelle uns helfen, zu verstehen, wie PK-Patienten mit halluzinatorischem Erleben umgehen (können).

Bereits früh wurde darauf hingewiesen, dass PK-Patienten problemorientierte und selbst entwickelte Copingstrategien (CS) erfolgreich nutzen [25]. Die Anwendung auf VH wurde in zwei Studien untersucht; hier wandten zwischen 53 und $78 \%$ der PK-Patienten CS an, die sie eigenständig oder unter klinischer Anleitung erprobt hatten ([5, 12]; • Tab. 2). CS können visueller, kognitiver oder interaktiver Art sein. So mag der Patient das halluzinierte Objekt nochmals aus nächster Nähe betrachten, um dann dessen Unwirklichkeit erkennen zu können. Er überprüft oder verbessert damit die visuelle Eingabe. Auch kann der nicht stattgefundene interne "Dialog" zwischen den zerebralen Netzwerken durch ein zwischenmenschliches Gespräch ersetzt werden (Frage an den/die Partner/in: „Siehst du dies auch?“). Hier zeigt sich also eine direkte Anwendbarkeit der multimodalen Erklärungsmodelle. Absichtlich werden auch andere sensorische Modalitäten aufgerufen (z.B. mit dem Stock das vermeintliche Objekt ertasten, den visuell Halluzinierten ansprechen und dann beruhigt sein, wenn keine Antwort erfolgt; [12]). Leider gibt es hierzu bisher nur die zwei erwähnten Beobachtungsstudien, jedoch keine kontrollierte Studie „Intervention" versus „keine Intervention". Wenn auch oft in kleinen Gruppen durchgeführt, gibt es hingegen zahlreiche Untersuchungen zum Einsatz kognitiver Verhaltenstherapie („,cognitive-behavioral treatment ${ }^{\prime \prime}$ ) bei akustischen Halluzinationen der Schizophrenie [60].

Es ist wahrscheinlich, dass aktive Kompensationsmechanismen - im Gegensatz 
Tab. 2 Kompensationsmechanismen der visuellen Halluzinationen bei der ParkinsonKrankheit

\begin{tabular}{l} 
Kognitive Strategien 53-69\% [5, 12] \\
$\begin{array}{l}\text { - Rationalisierung des Erlebten durch } \\
\text { Selbstreflektion }\end{array}$ \\
\hline Aktionsbasierte Strategien $65 \%[12]$ \\
- Erforschen des Ortes der VH mithilfe \\
anderer sensorischer Modalitäten (An- \\
sprechen, mit einem Stock betasten usw.)
\end{tabular}

zu einer Haltung des Negierens oder des „Für-sich-Behaltens" - die Lebensqualität der PK-Patienten verbessern [8]. Gewinnt der Patient früh und möglichst dauerhaft Einsicht in seine gestörte Wahrnehmung sowie den Umgang damit und wird er hierbei von Familienangehörigen aktiv unterstützt, so ist der Leidensdruck und das Stigma geringer, als wenn dies nicht geschieht. Auch kann man dann ggf. den Einsatz atypischer Neuroleptika hinauszögern oder ganz vermeiden. All dies setzt die regelmäßige Einbindung und Aufklärung der Angehörigen voraus, nicht zuletzt um zudem deren Stressniveau zu senken [55].

Der Vollständigkeit halber soll erwähnt werden, dass PK-Patienten selten einen Krankheitsgewinn aus dem VH-Erleben ziehen können. Wie eingangs angeführt, sehen einige Patienten $\mathrm{VH}$ als freudige Abwechslung in einem ansonsten trübem Alltag an [56]. Es wurde aber bisher nicht untersucht, ob PK-Patienten durch Eigensuggestion sog. eidetische Trugwahrnehmungen erleben können. Allerdings sind die meisten PK-Patienten der Ansicht, dass sie das VH-Erleben wenig beeinflussen oder gar steuern können [5].

\section{Diskussion und Ausblick}

Die Vielschichtigkeit möglicher Ursachen der VH bei der PK ist ausführlich dargestellt und erläutert worden. Es zeigt sich, dass die Erforschung der Ursachenkette sich zunehmend komplexen Netzwerkstörungen widmet. Einfachere Rezeptorhypothesen sind weitgehend verlassen worden. Auch wurde erörtert, dass es unterschiedliche Spielarten der VH gibt, mit wechselnder Ursachenverflechtung und variabler prognostischer Bedeutung. Vereinfachungen wie etwa: $\mathrm{VH}$ sind stets ein $\mathrm{Si}-$ gnum mali ominis oder VH können nur medikamentös angegangen werden, klingen zwar im klinischen Alltag verlockend, können aber auch zu vorschnellen prognostischen Aussagen und zum mangelhaften Ausschöpfen aller therapeutischen Möglichkeiten führen.

Es erscheint vielversprechend, in $\mathrm{Zu}-$ kunft die VH-Genese in Bezug aufFehlfunktionen des Visus und Störungen des Schlafs präziser zu erforschen. Es ist anzunehmen, dass damit auch Medikamente, die nicht auf das dopaminerge, sondern z. B. auf das cholinerge oder auf das serotoninerge System Einfluss nehmen, individueller oder „personalisiert" beim PK-Patienten mit VH zum Einsatz kommen können. Nicht zuletzt sollten Copingstrategien methodisch sauber erforscht werden, um damit Einzug in das therapeutische Routinearsenal zu finden.

\section{Fazit für die Praxis}

- Die Ursachenkette visueller Halluzinationen bei der PK ist komplexer als bisher angenommen.

- Dies erklärt z.B., warum nicht alle Patienten bei einer bestimmten Medikamentendosis visuelle Halluzinationen erleben.

- Besonders Visus- und Aufmerksamkeitsdefizite rücken ins Blickfeld.

- Funktionelle Magnetresonanztomographie kann fehlerhafte Netzwerkinteraktionen zeigen.

- Mit dem Patienten kann die Möglichkeit selbst anwendbarer Copingstrategien besprochen werden. Diese nichtmedikamentösen Behandlungsmethoden bedürfen aber noch weiterer wissenschaftlicher Validierung.

\section{Korrespondenzadresse}

\section{Prof. Dr. Nico J. Diederich}

Abteilung für Neurologie, Centre Hospitalier de Luxembourg

4, rue Barblé, 1210 Luxemburg-Stadt, Luxemburg

diederdn@pt.lu

Danksagung. Für die frühere Zusammenarbeit bei diesem Thema ist der Autor in Dankbarkeit verbunden mit Prof. Gilles Fénelon, PhD, MD, Hôpitaux Universitaires Henri Mondor, Créteil (Frankreich); Prof. CG Goetz, MD, Rush University Chicago (USA); Vannina Pieri, PhD, Centre Hospitalier de Luxembourg (Luxemburg) und Prof. Glenn Stebbins, PhD, Rush University Chicago (USA). Der Autor bedankt sich für die kritische Manuskriptdurchsicht bei Prof. A. Karenberg, Institut für Geschichte und Ethik der Medizin, Universität Köln und für die Anfertigung der Abbildungen 1 und 3 bei Frau Amanda Sidoli, Centre Hospitalier de Luxembourg.

Funding. Forschungszuwendungen: Fonds $\mathrm{Na}-$ tional de Recherche Luxembourg (FNR/06/04/05); Forschungsunterstützung durch: Fondation Think Luxembourg; Rotary International and Rotary Luxembourg; Beratertätigkeit: keine; Vortragshonorare: Alformec (Luxembourg)

\section{Einhaltung ethischer Richtlinien}

Interessenkonflikt. N.J. Diederich gibt an, dass kein Interessenkonflikt besteht.

Für diesen Beitrag wurden von den Autoren keine Studien an Menschen oder Tieren durchgeführt. Für die aufgeführten Studien gelten die jeweils dort angegebenen ethischen Richtlinien.

Open Access. Dieser Artikel wird unter der Creative Commons Namensnennung 4.0 International Lizenz veröffentlicht, welche die Nutzung, Vervielfältigung, Bearbeitung, Verbreitung und Wiedergabe in jeglichem Medium und Format erlaubt, sofern Sie den/die ursprünglichen Autor(en) und die Quelle ordnungsgemäß nennen, einen Link zur Creative Commons Lizenz beifügen und angeben, ob Änderungen vorgenommen wurden

Die in diesem Artikel enthaltenen Bilder und sonstiges Drittmaterial unterliegen ebenfalls der genannten Creative Commons Lizenz, sofern sich aus der Abbildungslegende nichts anderes ergibt. Sofern das betreffende Material nicht unter der genannten Creative Commons Lizenz steht und die betreffende Handlung nicht nach gesetzlichen Vorschriften erlaubt ist, ist für die oben aufgeführten Weiterverwendungen des Materials die Einwilligung des jeweiligen Rechteinhabers einzuholen.

Weitere Details zur Lizenz entnehmen Sie bitte der Lizenzinformation auf http://creativecommons.org/ licenses/by/4.0/deed.de. 


\section{Literatur}

1. Aarsland D, Larsen JP, Cummings JL et al (1999) Prevalence and clinical correlates of psychotic symptoms in Parkinson disease: a communitybased study. Arch Neurol 56:595-601

2. Archibald NK, Clarke MP, Mosimann UP et al (2011) Visual symptoms in Parkinson's disease and Parkinson's disease dementia. Mov Disord 26:2387-2395

3. Arnulf I, Bonnet AM, Damier P et al (2000) Hallucinations, REM sleep, and Parkinson's disease: a medical hypothesis. Neurology 55:281-288

4. Ballanger B, Strafella AP, van Eimeren T (2010) Serotonin 2A receptors and visual hallucinations in Parkinson disease. Arch Neurol 67:416-421

5. Barnes J, Connelly V, Boubert L et al (2013) Behavioural coping patterns in Parkinson's patients with visual hallucinations. J Neuropsychol 7:326-334

6. Bejr-Kasem H, Pagonabarraga J, Martínez-Horta S etal (2019) Disruption of the default mode network and its intrinsic functional connectivity underlies minor hallucinations in Parkinson's disease. Mov Disord 34:78-86

7. Bowman AR, Bruce V, Colbourn CJ et al (2017) Compensatory shifts in visual perception are associated with hallucinations in Lewy body disorders. Cogn Res Princ Implic 2:1-9

8. Bucks RS, Cruise KE, Skinner TC et al (2011) Coping processes and health-related quality of life in Parkinson's disease. Int J Geriatr Psychiatry 26:247-255

9. Collerton D, Perry E, McKeith I (2005) Why people see things that are not there: a novel perception and attention deficit model for recurrent complex visual hallucinations. Behav Brain Sci 28:737-757

10. Diederich NJ, Goetz CG, Raman R et al (1998) Poor visual discrimination and visual hallucinations in Parkinson's disease. Clin Neuropharmacol 21:289-295

11. Diederich NJ, Pieri V, Goetz CG (2000) Die optischen Halluzinationen des Parkinson-Patienten und das Charles Bonnet-Syndrom. Fortschr Neurol Psychiatr 68:129-136

12. Diederich NJ, Pieri V, Goetz CG (2003) Coping strategies for visual hallucinations in Parkinson's disease. Mov Disord 18:831-832

13. Diederich NJ, Goetz CG, Stebbins GT (2005) Repeated visual hallucinations in Parkinson's diseaseas disturbed external/internal perceptions: focused review and a new integrative model. Mov Disord 20:130-140

14. Diederich NJ, Goetz CG, Stebbins GT (2015) The pathology of hallucinations: one or several points of processing breakdown? In: Collerton D, Mosimann UP, Perry E (Hrsg) The neuroscience of visual hallucinations. Wiley-Blackwell, Hoboken, S 281-306 https://doi.org/10.1002/ 9781118892794

15. Dodel R, Koschel J, Lorenzl S et al (2018) Demenz mit Lewy-Körpern. Fortschr Neurol Psychiatr 86:43-47

16. Fénelon G, Mahieux F, Huon R et al (2000) Hallucinations in Parkinson's disease: prevalence, phenomenology and risk factors. Brain 123:733-745

17. Fénelon G, Goetz CG, Karenberg A (2006) Hallucinations in Parkinson disease in the prelevodopa era. Neurology 66:93-98

18. Ffytche D, Creese B, Politis M et al (2017) The psychosis spectrum in Parkinson disease. Nat Rev Neurol 13:81-95
19. Firbank MJ, Parikh J, Murphy N et al (2018) Reduced occipital GABA in Parkinson disease with visual hallucinations. Neurology 91:e675-e685

20. Forsaa EB, Larsen JP, Wentzel-Larsen T et al (2010) A 12-year population-based study of psychosis in Parkinson disease. Arch Neurol 67:996-1001

21. Gallagher DA, Parkkinen L, O'Sullivan SS et al (2011) Testing an etiological model of visual hallucinations in Parkinson's disease. Brain 134:3299-3309

22. Goetz CG, Stebbins GT (1995) Mortality and hallucinations in nursing home patients with advanced Parkinson's disease. Neurology 45:669-671

23. Goetz CG, Pappert EJ, Blasucci LM et al (1998) Intravenous levodopa in hallucinating Parkinson's disease patients: high-dose challenge does not precipitate hallucinations. Neurology 50:515-517

24. Haeske-Dewick HC (1995) Hallucinations in Parkinson's disease: characteristics and associated clinical features. Int JGeriatrPsychiatry 10:487-495

25. Haltenhof H, Krakow K, Zöfel P et al (2000) Krankheitsverarbeitung bei Morbus Parkinson. Nervenarzt 71:275-281

26. Han G, Han J, Han K et al (2020) Visual acuity and development of Parkinson's disease: a nationwide cohort study. Mov Disord 35:1532-1541

27. Harding AJ, Broe GA, Halliday GM (2002) Visual hallucinations in Lewy body disease relate to Lewy bodies in the temporal lobe. Brain 125:391-403

28. Haussmann R, Bauer M, Donix M (2016) Evidenzzur Behandlung der Parkinson-assoziierten Psychose. Nervenarzt 87:543-551

29. Hepp DH, Foncke EM, Olde Dubbelink KT et al (2017) Loss of functional connectivity in patients with Parkinson disease and visual hallucinations. Radiology 285:896-903

30. Jacobson SA, Morshed T, Dugger BN et al (2014) Plaques and tangles as well as Lewy-type alpha synucleinopathy are associated with formed visual hallucinations. Parkinsonism Relat Disord 20:1009-1014

31. Janzen J, van't Ent D, Lemstra AW et al (2012) The pedunculopontine nucleus is related to visual hallucinations in Parkinson's disease: preliminary results of a voxel-based morphometry study. J Neurol 259:147-154

32. Josephs KA (2007) Capgras syndrome and its relationship to neurodegenerative disease. Arch Neurol 64:1762-1766

33. Kalaitzakis ME, Christian LM, Moran LB et al (2009) Dementia and visual hallucinations associated with limbic pathology in Parkinson's disease. Parkinsonism Relat Disord 15:196-204

34. KassubekJ, DanekA, Del Tredici-BraakKetal (2013) Das Auge als Zugang zur Pathophysiologie von Parkinson-Syndromen. Nervenarzt 84:909-917

35. Kataoka H, Sugie K (2018) Delusional jealousy (Othello syndrome) in 67 patients with parkinson's disease. Front Neurol 7(9):129. https://doi.org/10. 3389/fneur.2018.00129

36. Lee JY, Kim JM, Ahn J et al (2014) Retinal nerve fiber layer thickness and visual hallucinations in Parkinson's disease. Mov Disord 29:61-67

37. Lefebvre S, Baille G, Jardri R et al (2016) Hallucinations and conscious access to visual inputs in Parkinson's disease. Sci Rep 6:36284. https://doi.org/10.1038/srep36284

38. Lenka A, Pagonabarraga J, Pal P et al (2019) Minor hallucinations in Parkinson disease: a subtle symptom with major clinical implications. Neurology 93:259-266

39. Lhermitte J (1922) Syndrome de la calotte pédonculaire. Les troubles psychosensoriels dans les lésions du mésencéphale. Rev Neurol (Paris) 38:1359-1365

40. Llorca PM, Pereira B, Jardri R et al (2016) Hallucinations in schizophrenia and Parkinson's disease: an analysis of sensory modalities involved and the repercussion on patients. Sci Rep 6:38152. https://doi.org/10.1038/srep38152

41. Manford M, Andermann F (1998) Complex visual hallucinations: clinical and neurobiological insights. Brain 121:1819-1840

42. Matar E, Phillips JR, Martens KAE et al (2019) Impaired colour discrimination-A specificmarker of hallucinations in Lewy body disorders. J Geriatr Psychiatry Neurol 32:257-264

43. Maurage CA, Ruchoux MM, de Vos R et al (2003) Retinalinvolvement in dementia with Lewybodies: a clue to hallucinations? Ann Neurol 54:542-547

44. Meppelink AM, de Jong BM, Renken R et al (2009) Impaired visual processing preceding image recognition in Parkinson's disease patients with visual hallucinations. Brain 132:2980-2993

45. Moser A, Hagenah J, Kömpf D (2003) Halluzinationen beim Morbus Parkinson. Nervenarzt 74:376-386

46. Mosimann UP, Rowan EN, Partington CEetal (2006) Characteristics of visual hallucinations in Parkinson disease dementia and dementia with Lewy bodies. Am J Geriatr Psychiatry 14:153-160

47. Moskovitz C, Moses H, Klawans HL (1978) Levodopa-induced psychosis: a kindling phenomenon. Am J Psychiatry 135:669-675

48. Nebe A, Ebersbach G (2007) Selective diplopia in Parkinson's disease: a special subtype of visual hallucination? Mov Disord 22:1175-1178

49. Onofrj M, Bonanni L, Albani G et al (2006) Visual hallucinations in Parkinson's disease: clues to separate origins. J Neurol Sci 248:143-150

50. Onofrj M, Espay AJ, Bonanni L et al (2019) Hallucinations, somatic-functional disorders of PD-DLB as expressions of thalamic dysfunction. Mov Disord 34:1100-1111

51. Pagonabarraga J, Martinez-Horta S, Fernández de Bobadilla Ret al (2016) Minor hallucinations occur in drug-naive Parkinson's disease patients, even from the premotor phase. Mov Disord 31:45-52

52. Perry EK, Perry RH (1995) Acetylcholine and hallucinations: disease-related compared to druginduced alterations in human consciousness. Brain Cogn 28:240-258

53. Pieri V, Diederich NJ, Raman R et al (2000) Decreased colour discrimination and contrast sensitivity in Parkinson's disease. J Neurol Sci 172:7-1

54. Ramírez-Ruiz B, Martí MJ, Tolosa E et al (2007) Cerebral atrophy in Parkinson's disease patients with visual hallucinations. Eur J Neurol 14:750-756

55. Renouf S, Ffytche D, Pinto R et al (2018) Visual hallucinations in dementia and Parkinson's disease: a qualitative exploration of patient and caregiver experiences. Int J Geriatr Psychiatry 33:1327-1334

56. Sacks O (2012) Hallucinations. Pan Macmillian, London

57. Seidel K, Mahlke J, Siswanto S et al (2015) The brainstem pathologies of Parkinson's disease and dementia with Lewy bodies. Brain Pathol 25:121-135

58. Shine JM, Halliday GM, Naismith SL et al (2011) Visual misperceptions and hallucinations in Parkinson's disease: dysfunction of attentional control networks? Mov Disord 26:2154-2159

59. Teunisse RJ, Zitman FG, Cruysberg JRM et al (1996) Visual hallucinations in psychologically 
normal people:Charles Bonnet's syndrome. Lancet 347:794-797

60. van der Gaag M, Valmaggia LR, Smit F (2014) The effects of individually tailored formulationbased cognitive behavioral therapy in auditory hallucinations and delusions: a meta-analysis. Schizophr Res 156:30-37

61. Visser F, Vlaar AMM et al (2019) Diplopia in Parkinson's disease: visual illusion or oculomotor impairment? J Neurol 266:2457-2464

62. Walpola IC, Muller AJ, Hall JM et al (2020) Mindwandering in Parkinson's disease hallucinations reflects primary visual and default network coupling. Cortex 125:233-245

63. Weil RS, Schrag AE, Warren JD et al (2016) Visual dysfunction in Parkinson's disease. Brain 139:2827-2843

64. Wood RA, Hopkins SA, Moodley KK et al (2015) Fifty percent prevalence of extracampinehallucinations in Parkinson's disease patients. Front Neurol 6:263. https://doi.org/10.3389/fneur.2015.00263

65. Zarkali A, Lees AJ, Weil RS (2019) Flickering stimuli do not reliably induce visual hallucinations in Parkinson's disease. J Parkinsons Dis 9:631-635

\section{Causes of visual hallucinations in Parkinson's disease}

Background: Visual hallucinations (VH) have mainly been considered as late symptoms of Parkinson's disease (PD); however, minor forms of $\mathrm{VH}$ also occur in early stages of the disease. Initially dopaminergic overstimulation was discussed as the cause and later on VH have been considered as an early red flag of dementia in PD.

Objective: The present study analyzed whether the pathophysiological concept of $\mathrm{VH}$ has been enlarged in recent years.

Material and methods: Clinical, pharmacological, neuropathological as well as functional magnetic resonance imaging studies dealing with VH were reviewed. A systematic classification in monomodal and multimodal models of $\mathrm{VH}$ is proposed. The applicability to various forms of $\mathrm{VH}$ and various triggering situations is critically examined.

Results: Reduction of the visual information input, erroneous visual processing, attention deficits, and dysfunctional connectivity between various cerebral networks have been shown. There is partial overlapping with the Lhermitte syndrome and the Charles Bonnet syndrome. No model is able to fully explain all VH variants. Not all VH have the same pathogenesis and the same poor prognosis.

Conclusion: The chain of causes underlying VH is complex and can vary from patient to patient. So far the therapeutic applications are largely unexplored; however, there is preliminary evidence that beside adjustment of the medication, improvement of visual acuity, active involvement of the partner, and possibly, individually adaptable coping strategies could be successfully implemented.

\section{Keywords}

Parkinson's disease - Visual hallucinations - Charles Bonnet syndrome - Lhermitte syndrome . Coping strategies 